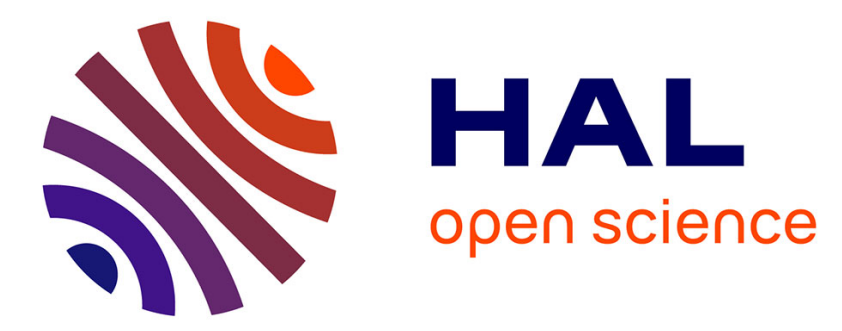

\title{
Optimal Pattern Generator For Dynamic Walking in humanoid Robotics
}

David Galdeano, Ahmed Chemori, Sébastien Krut, Philippe Fraisse

\section{To cite this version:}

David Galdeano, Ahmed Chemori, Sébastien Krut, Philippe Fraisse. Optimal Pattern Generator For Dynamic Walking in humanoid Robotics. SSD: Systems, Signals and Devices, Mar 2013, Hammamet, Tunisia. 10.1109/SSD.2013.6564107 . lirmm-00804250

\section{HAL Id: lirmm-00804250 https://hal-lirmm.ccsd.cnrs.fr/lirmm-00804250}

Submitted on 25 Mar 2013

HAL is a multi-disciplinary open access archive for the deposit and dissemination of scientific research documents, whether they are published or not. The documents may come from teaching and research institutions in France or abroad, or from public or private research centers.
L'archive ouverte pluridisciplinaire HAL, est destinée au dépôt et à la diffusion de documents scientifiques de niveau recherche, publiés ou non, émanant des établissements d'enseignement et de recherche français ou étrangers, des laboratoires publics ou privés. 


\title{
Optimal Pattern Generator For Dynamic Walking in humanoid Robotics
}

\author{
David Galdeano, Ahmed Chemori, Sebastien Krut and Philippe Fraisse \\ LIRMM, Université Montpellier 2 \\ LIRMM - UMR 5506 - CC 477, 161 rue Ada, 34095 Montpellier Cedex 5, France. \\ Email: galdeano(chemori,krut,fraisse)@lirmm.fr
}

\begin{abstract}
This paper deals with an optimal ZMP based pattern generator for stable dynamic walking. The proposed method is based on a Three-Mass Linear Inverted Pendulum Model (3MLIPM), used as a simplified dynamics of the biped robot. The 3MLIPM simplifies the biped robot as a three point masses and two-link system. A ZMP based criterion is then used in an optimization problem whose solution gives the best values of the model's parameters w.r.t. dynamic walking stability. Numerical simulations are presented to show the effectiveness of the proposed optimal pattern generator.
\end{abstract}

\section{INTRODUCTION}

A humanoid robot is a robot with an appearance based on that of the human body. Humanoid robot walking is a very challenging field of research due to the complexity of synthesizing stable walking gaits for these systems. Within this field, humanoid robot control needs sophisticated control schemes to deal with their complexity including:

- nonlinear and high-order dynamics,

- variable structure model according to the different phases of the walking cycle,

- contact constraints with the ground that should be managed,

- the hybrid character of the dynamics due to rigid impacts between the robot's foot and the ground,

- stability during walking that should be ensured.

Most of the proposed control schemes in the literature are based on the use of reference trajectories that should be followed in real time. This fact shows the importance of a pattern generator in humanoid walking control. Several types of pattern generators have then been proposed. However, those who guarantee a-priori stability are often based on one of the following stabilization criteria:

- The Center Of Mass (COM)

- The Zero Moment Point (ZMP)

- The Foot Rotation Indicator (FRI)

The COM [1] is the mean location of all masses of the robot links. It is usually used as a static stability criterion.

The ZMP [2], [3] is the point of junction between the vertical reaction forces and the ground. It is the most used dynamic stability criterion.

The FRI [4] is a point on the foot/ground-contact surface where the net ground-reaction force would have to act for the aim to keep the foot stationary. It is also used as a dynamic stability criterion.

This research was supported by the project R2A2 (ANR- 09-SEGI-011).

\section{RELATED WORK}

In the literature several methods have been proposed for generation of walking trajectories in humanoid robotics.

One interesting method is the so called Inverted Pendulum Model (IPM) [5], [6] that considers the robot as a single point mass and massless legs. This method simplifies the dynamics of the robot to an inverted pendulum with a pointwise mass linked by a telescopic leg to a spherical ground/leg joint.

The Linear Inverted Pendulum Model (LIPM) [7], [8] is an extension of the IPM were the height of the torso is kept constant leading to a more natural movement.

The IPM considers the humanoid robot as an inverted pendulum with a pointwise mass and ignores the dynamics of the legs. This fact can involve a loss of walking stability since the considered model is not sufficiently accurate. For instance, this is the case when the robot legs are heavy or without a torso (case of our robot SHERPA). In order to obtain a more accurate model for these walking robots, it would be necessary to consider the dynamics of the legs by using a preview control to correct the error between the IPM and the real robot as in [9] or to use a multiple pointwise mass model instead of considering a one pointwise mass model.

For instance, a Two-Mass Inverted Pendulum Model (TMIPM) and Multiple Mass Inverted Pendulum Model (MMIPM) extending the IPM were studied in [10]. In the MMIPM, the considered model is composed by more than one mass. For instance these masses are located at the hip and along the swinging leg in the case of a two-mass model.

The Gravity-Compensated Inverted Pendulum Model (GCIPM) [11] uses one mass to represent the body of the robot and an additional mass to represent the swing leg.

A three-mass model using the concept of ZMP has been studied in [12], however it doesn't use any LIPM to generate trajectories and the torso of the biped robot moves up and down.

A generation of walking trajectories using a three pointwise mass model to compute reference torque instead of ZMP trajectories has been proposed in [13].

An other three-mass model using the inverted pendulum concept is that proposed in [14]. However, the geometrical parameters, such as position and location of masses, have been chosen arbitrarily. One good idea would be to tune this parameters at their best values in order to enhance the walking stability of the walking robot, and this is the scope of the present paper.

The proposed solution is an extension of the method proposed in [14] to deal with dynamic stability of walking in 
the generated trajectories, as well as change of direction during walking. The robot's dynamics is approximated by a Three-Mass Linear Inverted Pendulum. The hip and feet trajectories are generated by the movement of masses. The walking movement of the robot is generated in the sagittal and frontal planes separately. The joint trajectories are computed using inverse kinematics of the biped robot. In order to ensure a stable dynamic walking, the parameters of the model must be well tuned. The best way is to perform this tuning through an optimization of the generated joints' trajectories to minimize the the ZMP excursion within the footprint. The second contribution of this paper is the online change of walking direction, where new optimal trajectories are generated to ensure a-priori stability during walking while turning.

This paper is organized as follows: in next section, the prototype of our demonstrator SHERPA is introduced. Section IV introduces the simplified model that will be used in the generation of reference trajectories, as well as the pattern generator proposed in [14]. In section V, an extension of this method is presented, where our contributions are highlighted. Numerical simulations are presented and discussed in Section VI where the effectiveness of the proposed method is shown and compared with the original method. The paper ends with some concluding remarks.

\section{DESCRIPTION OF SHERPA PROTOTYPE}

The SHERPA walking robot (cf. Fig. 1) is a French biped robot developed at LIRMM laboratory (http://www.lirmm.fr) within the framework of the national project ANR-BLANC SHERPA. This robot is built to carry loads while walking in a human environment [15].

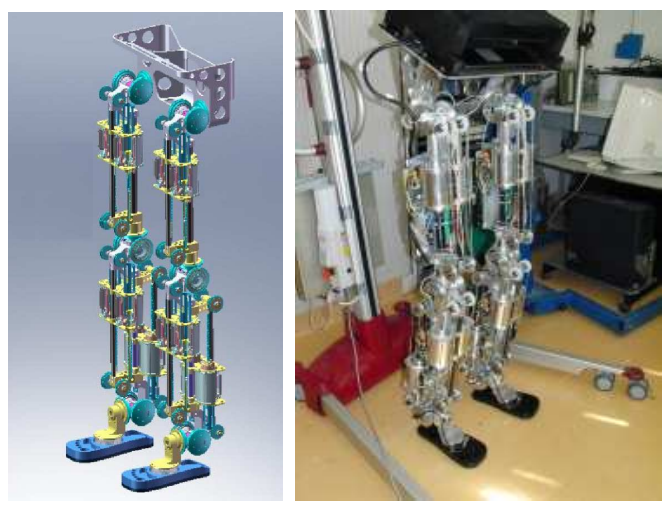

Fig. 1. The SHERPA biped robot

The SHERPA biped robot is composed of one waist linking two legs together. Each leg has six degrees of freedom (dof), and the robot is equiped with 12 actuators, which is enough to reproduce a human walking.

These dofs are distributed on different articulations of the robot as follows: three dofs at the hip, one dof at the knee and two dofs at the ankle.

The geometric parameters of SHERPA are summarized in TABLE I.

The computation of the ZMP and CoM of the robot is based on the formalism presented in [16], in which the ZMP evaluation uses the angular momentum.

\begin{tabular}{|c|l|c|}
\hline Parameter & Description & Value \\
\hline$L_{\text {shin }}$ & Length of shin & $0.51 \mathrm{~m}$ \\
\hline$L_{\text {thigh }}$ & Length of thigh & $0.5033 \mathrm{~m}$ \\
\hline$L_{s}$ & Step width & $0.31 \mathrm{~m}$ \\
\hline mass & Weight of the robot & $47.1 \mathrm{~kg}$ \\
\hline
\end{tabular}

TABLE I

GEOMETRIC PARAMETERS OF SHERPA BIPED ROBOT

\section{Three-Mass Linear Inverted Pendulum Model}

The three-mass Linear Inverted Pendulum Model (3MLIPM) as introduced in [14] simplifies the biped robot to a three links system (cf. Fig. 2) with a pointwise mass on each link. The three masses represent the torso and the two legs, unlike the single mass model (as in IPM or LIPM) where a unique mass is located at the hip of the robot. The links are connected together at the hip.

This model is more accurate than the LIPM, especially for a biped robot without torso, where the position of the CoM can be very different from the hip position.

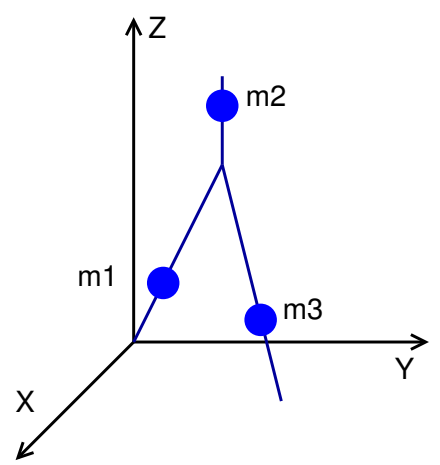

Fig. 2. Graphical representation of the Three-Mass Linear Inverted Pendulum Model

From Fig. 2, the equation of moment applied on the supporting ankle can be formulated as follows:

$$
\begin{aligned}
\tau_{x} & =\sum_{i=1}^{3} m_{i}\left(g+\ddot{z}_{i}\right) y_{i}-\sum_{i=1}^{3} m_{i} \ddot{y}_{i} z_{i} \\
\tau_{y} & =\sum_{i=1}^{3} m_{i} \ddot{x}_{i} z_{i}-\sum_{i=1}^{3} m_{i}\left(g+\ddot{z}_{i}\right) x_{i}
\end{aligned}
$$

where $\tau_{x}$ and $\tau_{y}$ are the torques applied on the ankle, $m_{i}$ represents the value of the $i^{t h}$ pointwise mass, $\left(x_{i}, y_{i}, z_{i}\right)$ are the Cartesian positions of the $i^{t h}$ pointwise mass $(i=1,2$ or 3 represents respectively the stance leg, the torso and the swing leg).

For simplification, the height of the masses can be assumed constant and the torque applied on the supporting ankle is assumed to be zero [14]. With these assumptions, (1) and (2) can be simplified into:

$\sum_{i=1}^{3} m_{i} \ddot{y}_{i} z_{i}=\sum_{i=1}^{3} m_{i} g y_{i}$ and $\sum_{i=1}^{3} m_{i} \ddot{x}_{i} z_{i}=\sum_{i=1}^{3} m_{i} g x_{i}$

These equations are decoupled, so the movement can be generated in the sagittal and lateral planes separately. 


\section{A. Assumptions and notations}

The following assumptions are considered to simplify calculations [14]:

- The ground is flat and horizontal.

- The height variation of each mass can be neglected.

- The double support phase is considered instantaneous.

- There is no energy loss during impact.

- The standing foot has no overturn when touching and leaving the ground.

- The swing foot is parallel to the ground.

- The torso is upright.

The following notations are used:

- 2T: The whole stepping cycle.

- $2 D_{s}$ : The steeping length.

- $L_{s}$ : The width of a step.

B. Motion in sagittal plane

The movement in the sagittal plane and the associated notations are illustrated in Fig. 3.

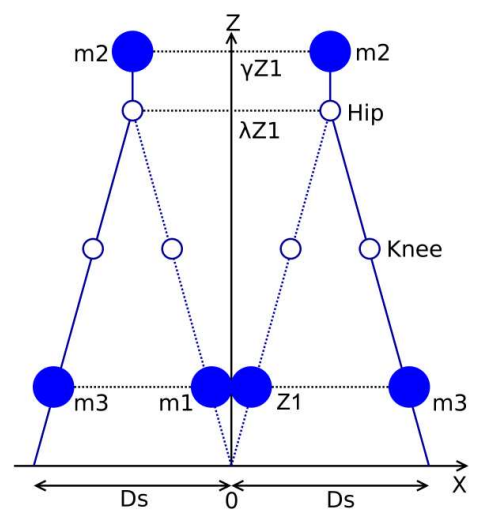

Fig. 3. Illustration of 3MLIPM in the sagittal plane

With the above assumptions, heights and masses can be normalized as follow:

$$
z_{2}=\gamma z_{1} \quad z_{3}=z_{1} \quad x_{2}=\lambda x_{1} \quad m_{2}=k m_{1} \quad m_{3}=m_{1}
$$

were $\gamma, \lambda$ and $k$ are parameters extracted from the robot's model for computing. With (4), (3) can be simplified into:

$$
\begin{gathered}
b \ddot{x}_{1}+d \ddot{x}_{3}=a x_{1}+x_{3} \\
a=k \lambda+1 \quad, \quad b=\frac{z_{1}}{g}(1+k \gamma \lambda) \quad, \quad d=\frac{z_{1}}{g}
\end{gathered}
$$

In order to generate walking gaits, a trajectory for the swing foot $\left(m_{3}\right)$ is necessary. If the trajectory of this last one is known, then those of the two other masses can be computed. According to [14], a sinusoidal function is chosen to represent the trajectory of the mass $m_{3}$, as follows:

$$
x_{3}=k \cos (\omega t+\phi)
$$

were parameters $\phi$ and $k$ are given in (11). Here, $\omega$ is not the step frequency, it's a parameter that must be chosen to keep the foot position and velocity positive in order to ensure a forward movement of the foot during the step.

Using (5) and (7), $x_{1}$ can be expressed by:

$$
x_{1}=C_{1} \cos (\omega t+\phi)+C_{2} e^{\sqrt{\frac{a}{b}} t}+C_{3} e^{-\sqrt{\frac{a}{b}} t}
$$

With the above assumptions given in sectionIV-A, initial conditions can be fixed as:

$$
\begin{gathered}
x_{1}(0)=-\frac{D_{s}}{2 \lambda} \quad, \quad x_{3}(0)=-\frac{D_{s}}{2 \lambda}(2 \lambda-1) \\
x_{1}\left(\frac{T}{2}\right)=0 \quad, \quad x_{3}\left(\frac{T}{2}\right)=0
\end{gathered}
$$

The equations' coefficients can be found using initial conditions:

$$
\begin{array}{r}
\phi=-\frac{\pi}{2}-\frac{T}{2} \omega \quad, \quad k=-\frac{D_{s}(2 \lambda-1)}{2 \lambda \cos (\phi)} \\
C_{1}=-\frac{k\left(1+d \omega^{2}\right)}{a+b \omega^{2}} \quad C_{3}=-e^{\sqrt{\frac{a}{b}} t} C_{2} \\
C_{2}=\frac{1}{1-e^{\sqrt{\frac{a}{b}} t}}\left(-\frac{D_{s}}{2 \lambda}-C_{1} \cos (\phi)\right)
\end{array}
$$

With all pointwise masses trajectories computed, the trajectories of hip and ankle can then be determined using geometrical restrictions from Fig. 3.

$$
\begin{array}{ll}
x_{s t}(t)=0 \text { for } 0 \leq t \leq T, x_{h}(t)=\lambda x_{1}(t) & \text { for } 0 \leq t \leq T \\
z_{s t}(t)=0 \text { for } 0 \leq t \leq T, & z_{h}(t)=\lambda z_{1} \quad \text { for } 0 \leq t \leq T
\end{array}
$$

where $x_{s t}, z_{s t}$ are the positions of the stance foot along $x$ and $z$ axis respectively, and $x_{h}, z_{h}$ are the positions of the hip along $x$ and $z$ axis respectively.

The vertical position of the swing foot needs to be higher than the floor during walking. A sinusoidal shape is then proposed for the swing foot trajectory along the $z$ axis. Due to previous assumptions, the influence of this change of height is neglected in computations.

$$
\begin{array}{ll}
x_{s w}(t)=\frac{\lambda x_{3}(t)-\lambda x_{h}(t)}{\lambda-1} & \text { for } 0 \leq t \leq T \\
z_{s w}(t)=\frac{\sqrt{2} H_{s}}{2}\left(\sin \left(\frac{2 \pi}{T} t-\frac{\pi}{2}\right)+1\right)^{0.5} & \text { for } 0 \leq t \leq T
\end{array}
$$

where $x_{s w}, z_{s w}$ are the positions of the swing foot along $x$ and $z$ axis respectively, and $H_{s}$ is the maximal height of the swing foot during walking.

\section{Motion in frontal plane}

The movement in the frontal plane and the associated notations are illustrated in Fig. 4 below.

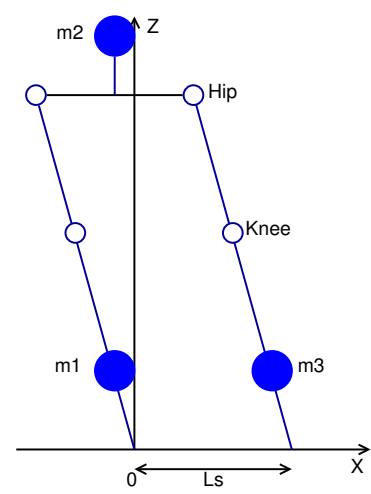

Fig. 4. Illustration of 3MLIPM in the frontal plane 
The 3MLIPM equations are now applied in the frontal plane.

$$
\begin{gathered}
u \ddot{y}_{1}-v y_{1}=w \\
u=(2+k \lambda \gamma) \frac{z_{1}}{g}, v=(2+k \lambda), w=\left(L_{s}+k \frac{L_{s}}{2}\right)
\end{gathered}
$$

These equations are solved considering the following initial conditions:

$$
y_{1}(0)=0 \quad, \quad \dot{y}_{1}\left(\frac{T}{2}\right)=0 \quad, \quad y_{1}(T)=0
$$

The trajectory of the mass $m_{1}$ along the $y$ axis is then computed and expressed by:

$$
\begin{gathered}
y_{1}(t)=C_{1} e^{\sqrt{\frac{v}{u}} t}+C_{2} e^{-\sqrt{\frac{v}{u}} t}-\frac{w}{v} \\
C_{1}=\frac{w}{v} \frac{1-e^{\sqrt{\frac{v}{u}} T}}{1-e^{2 \sqrt{\frac{v}{u}} T}} \quad, \quad C_{2}=e^{\sqrt{\frac{v}{u}} t} C_{1}
\end{gathered}
$$

From the trajectory of $m_{1}$, the trajectories of $m_{2}$ and $m_{3}$ can be determined using geometrical restrictions illustrated on Fig. 4. From these trajectories and the inverse kinematics of the robot, the trajectories of joints can then easily be computed.

\section{Adaptation of the general model for SHERPA robot}

The SHERPA robot is a biped robot without a torso (cf. Fig. 1). In order to simplify the model, the torso pointwise mass is set on the hip. This change results in $\gamma=\lambda$.

The basic principle of proposed method in [14] and summarized above uses geometrical parameters of the robot but doesn't give a method to calculate $z_{1}$ and $m_{1}$. In order to take into account the dynamic stability of the resulting walking trajectories, these parameters are proposed to be tuned using optimization to enhance this walking performance.

\section{REFERENCE TRAJECTORIES OptIMIZATION}

The proposed pattern generator in [14] computes joint reference trajectories using arbitrary defined parameters. This can be improved by choosing the best values of these parameters to ensure the dynamic walking stability. The position of the Zero Moment Point (ZMP) [2] is then used during the walking as an indicator of stability.

\section{A. Optimization w.r.t. dynamic walking stability}

The first main contribution of this paper is to improve the pattern generator proposed in [14] by using an optimization criterion in order to find the best values for $z_{1}$ and $m_{1}$ to enhance the dynamic walking stability.

In order to improve such a stability, the ZMP corresponding to the generated joint trajectories should be computed with a full robot dynamics and compared with the desired ZMP, set to the center of the stance foot. This chosen desired position corresponds to a maximum stability margins.

The following objective function is then proposed to be optimized w.r.t. parameters $z_{1}$ and $m_{1}$ :

$$
\left[\begin{array}{c}
\hat{z_{1}} \\
\hat{m}_{1}
\end{array}\right]=\underset{\left[\begin{array}{c}
z_{1} \\
m_{1}
\end{array}\right]}{\operatorname{ArgMinMax}\left(\sqrt{\frac{1}{\alpha}\left(x_{z m p}-x_{d z m p}\right)^{2}+\frac{1}{\beta}\left(y_{z m p}-y_{d z m p}\right)^{2}}\right)}
$$

where $x_{z m p}$ and $y_{z m p}$ are the positions of computed ZMP, $x_{d z m p}$ and $y_{d z m p}$ are those of the desired one. $z_{1}$ and $m_{1}$ are the optimization parameters, $\alpha$ and $\beta$ are the length and the width of the foot (i.e. along $x$ axis and $y$ axis respectively, cf Fig. 5).

By using a normalized deviation of the ZMP with respect to the center of the footprint of the stance foot as cost function, the objective function computes the mass distribution to ensure the largest stability margins.

\section{B. Change of direction and stability optimization}

One classical feature of pattern generator is to allow the biped robot to change walking direction like in [17]. As proposed in [14], the 3MLIPM pattern generator is designed to make the robot walk in a straight line, our second contribution is then to modify the original 3MLIPM to allow a change of direction while the biped robot is walking. The change of direction is obtained by applying a rotation at the hip of the stance leg, as follows:

$$
\Omega=-\frac{R}{2} \cos \left(\frac{\pi t}{T}\right)
$$

with $\Omega$ is the angle of rotation and $R$ is its amplitude.

The change of direction alters the stability of the biped robot. Again, the proposed optimization criterion (19) is used to improve dynamic walking stability. The solution of this optimization problem gives the best values for $z_{1}$ and $m_{1}$ which allow a better dynamic stability of the robot walking and turning.

\section{Stability margins}

The stability margins are defined as illustrated in Fig. 5.

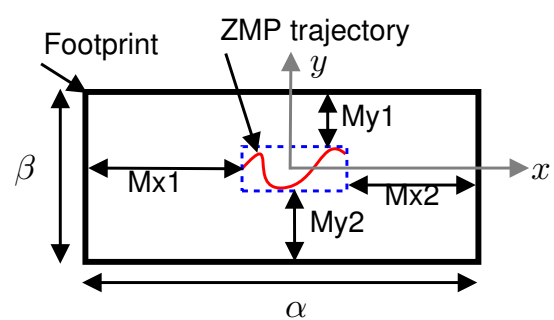

Fig. 5. ZMP displacement under the footprints and stability margins

The dashed interior rectangle explicit the boundaries of the ZMP displacements. Based on Fig. 5, the stability margins can be computed as follows:

$$
\begin{aligned}
& M x_{1}=\frac{\alpha}{2}+\operatorname{Min}\left(d Z M P_{x}(t)\right), M x_{2}=\frac{\alpha}{2}-\operatorname{Max}\left(d Z M P_{x}(t)\right) \\
& M y_{1}=\frac{\beta}{2}+\operatorname{Min}\left(d Z M P_{y}(t)\right), M y_{2}=\frac{\beta}{2}-\operatorname{Max}\left(d Z M P_{y}(t)\right) \\
& \text { for all } t \in\left[t s_{i}, t s_{f}\right] \\
& M x=\operatorname{Min}\left(M x_{1}, M x_{2}\right), M y=\operatorname{Min}\left(M y_{1}, M y_{2}\right)
\end{aligned}
$$

were $d Z M P_{x}(t)$ and $d Z M P_{y}(t)$ are the deviation of the ZMP trajectory with respect to the center of the stance foot along $x$ axis and $y$ axis respectively, $t s_{i}$ and $t s_{f}$ are times of landing and lift-off of the stance foot respectively. 


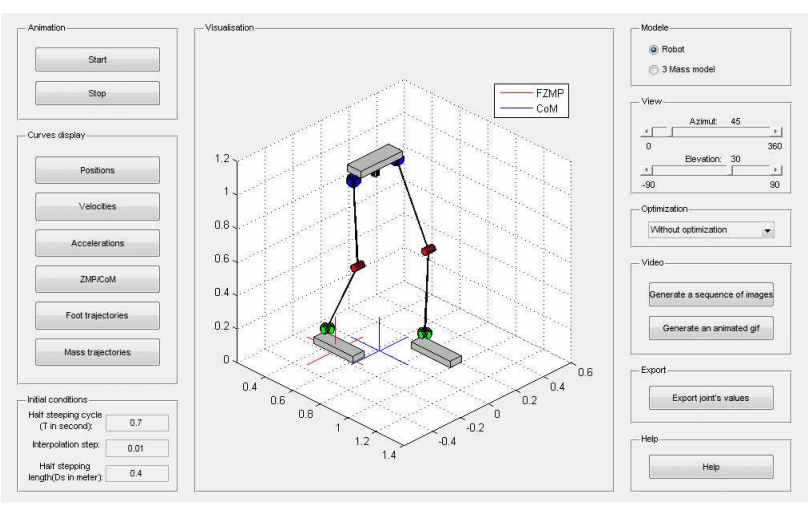

Fig. 6. Graphical User Interface of SHERPA biped robot simulator

\section{Simulation Result}

A simulator for SHERPA biped robot was developed using the Graphical User Interface of Matlab ${ }^{\mathrm{TM}}$ software. The graphical interface of this simulator is shown in Fig. 6.

This interface enables to tune some parameters of the robot as well as those of the optimization criterion. A graphical animation of the robot or the three mass model can also be displayed to show the obtained movements.

The parameters $z_{1}$ and $m_{1}$ are computed by optimization, the other parameters are constant and summarized in TABLE II.

\begin{tabular}{|c|l|l|}
\hline Parameter & Description & Value \\
\hline$m_{1}$ & Weight of one leg & Calculated \\
\hline$m_{2}$ & Weight of the torso & mass $-2 m_{1}$ \\
\hline$z_{1}$ & Height of the mass $m_{1}$ & Calculated \\
\hline$z_{2}$ & Height of the hip & $\left(L_{s h i n}+L_{\text {thigh }}\right) \cdot 0.95$ \\
\hline$T$ & Step time & $0.7 \mathrm{~s}$ \\
\hline$D_{s}$ & Step length & $0.4 \mathrm{~m}$ \\
\hline$\omega$ & Calculus variable & $\pi /(5 \cdot T)$ \\
\hline$\lambda=\gamma$ & Calculus variable & $z_{2} / z_{1}$ \\
\hline
\end{tabular}

TABLE II

PARAMETERS OF SHERPA BIPED ROBOT

Using the developed simulator, three simulation scenarios are proposed to validate the proposed optimal pattern generator, namely:

- optimal trajectories generation for straight walking,

- trajectories generation for walking with change of direction without optimisation,

- trajectories generation for walking with change of direction and optimization.

These simulations will be detailed in the following.

\section{A. Simulation 1: Straight walking}

The optimization criterion given in (19) is used to find the best values for $z_{1}$ and $m_{1}$ using the fminsearch algorithm proposed within Matlab software. The obtained optimal solution is summarized in table III.

\begin{tabular}{|c|l|l|}
\hline Parameter & Without optimization & With optimization \\
\hline$z_{1}$ & $0.6 \mathrm{~m}$ & $0.2598 \mathrm{~m}$ \\
\hline$m_{1}$ & $6 \mathrm{~kg}$ & $0.4442 \mathrm{~kg}$ \\
\hline
\end{tabular}

TABLE III

RESULTING OPTIMAL MASS DISTRIBUTION

The obtained simulation results are illustrated through Figs. 7-10.

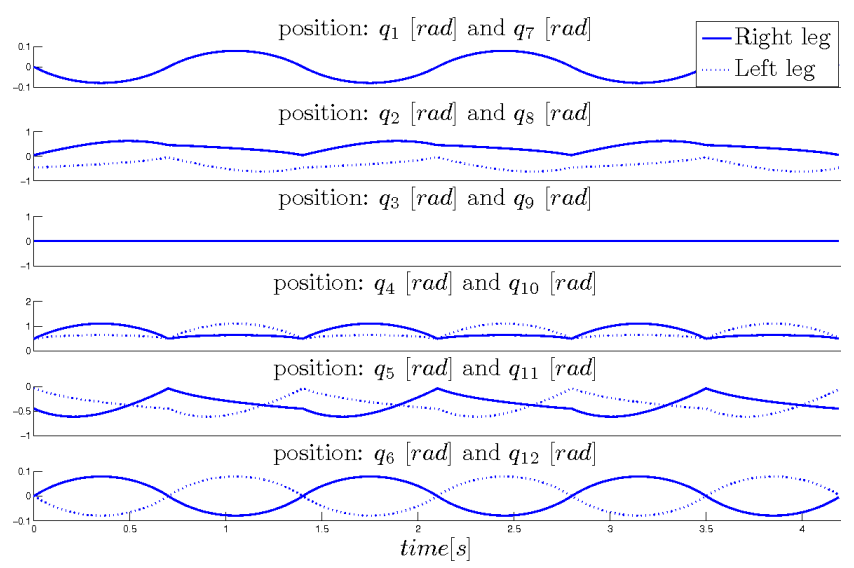

Fig. 7. Joints' positions with optimization ZMP

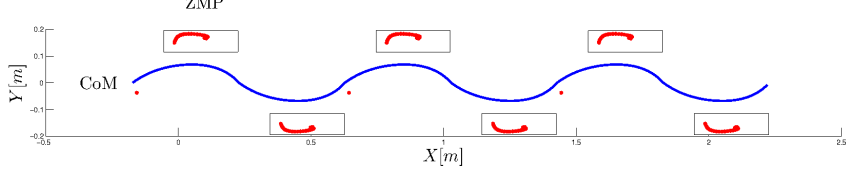

Fig. 8. Evolution of ZMP and CoM trajectories with the LIPM

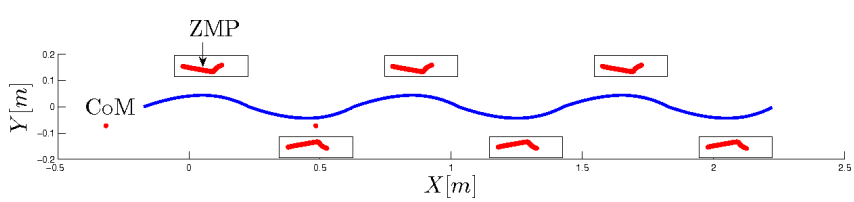

Fig. 9. Evolution of ZMP and CoM trajectories with original 3MLIPM

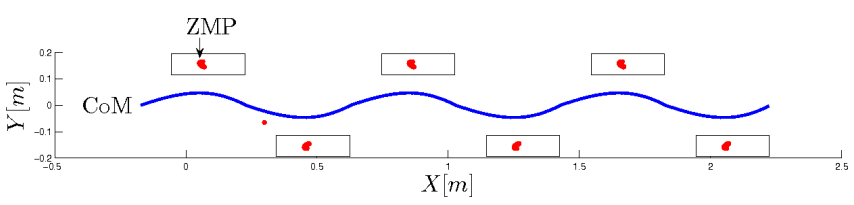

Fig. 10. Evolution of ZMP and CoM trajectories with optimal 3MLIPM

Figure 7 represents the evolution of the joints' positions, where it can clearly be seen that the obtained trajectories are periodic.

Figures 8, 9 and 10 represent the evolution of the ZMP position, and the CoM position with respect to footprints of the biped robot on the ground using respectively the linear inverted pendulum model (LIPM), the original (3MLIPM) pattern generator proposed in [14] and the optimal one proposed in this paper.

For the LIPM as well as the original 3MLIPM, the ZMP moves inside the footprint with a big variation, however when the optimal parameters are used, the ZMP is more concentrated in the center of the footprint. Therefore, the walking dynamic stability is clearly improved with the proposed optimal 3MLIPM pattern generator due to the increase of stability margins.

\section{B. Simulation 2: Walking with change of direction}

The objective of this simulation is to evaluate the stability margins of the pattern generator proposed in [14] with a change of direction during walking. It is worth to note that the change of direction during walking has not been yet considered 
in the original 3MLIPM, therefore a simple simulation (cf. Fig. 11) shows the loss of stability during dynamic walking.

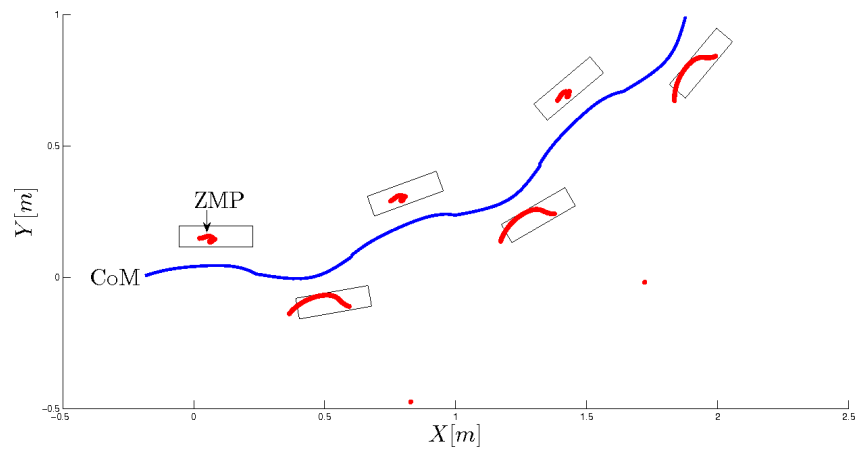

Fig. 11. Evolution of ZMP and CoM trajectories with change of direction

Indeed, when the robot changes the walking direction, the position of ZMP is moving within footprints, being sometimes outside of the stance foot, therefore the robot becomes unstable. A computation of optimal parameters in this scenario is necessary to improve the dynamic walking stability. This will be the objective of next simulation scenario.

\section{Simulation 3: Change of direction with optimization}

In the proposed optimal pattern generator, the optimization process computes the best values for parameters $z_{1}$ and $m_{1}$ and uses them to generate the joints' trajectories.

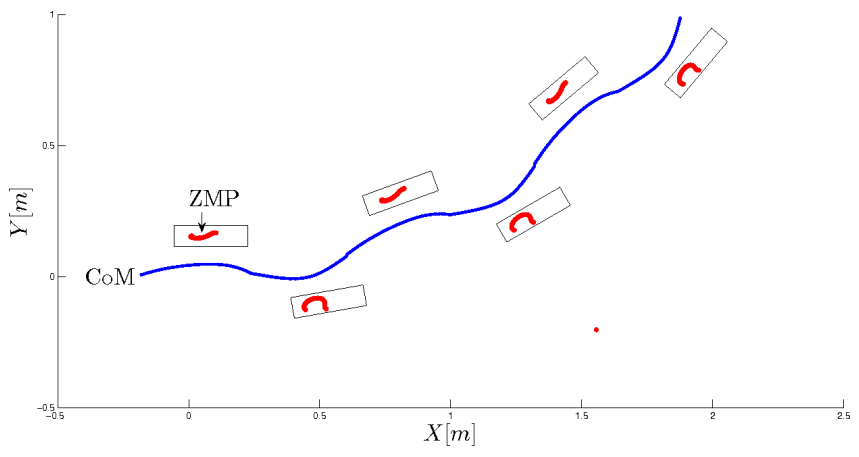

Fig. 12. Evolution of ZMP and CoM trajectories with change of direction and optimization

Figure 12 represents the evolution of the ZMP and the CoM positions as well as footprints of the biped robot on the ground level when turning with an angle of 20 degrees by step with the obtained optimal values of $z_{1}$ and $m_{1}$.

With this optimization, according to Fig. 12, the robot remains stable during walking while turning since it keeps the ZMP always inside the footprint of the supporting leg.

According to the obtained results, from simulation 1 and 3 , it is clearly shown that the proposed optimal pattern generator has significantly improved the dynamic stability of the biped robot.

\section{COnClusions And Future Works}

The objective of this work was to generate stable dynamic walking for SHERPA biped robot. To reach this objective, the original pattern generator proposed in [14] based on a ThreeMass Linear Inverted Pendulum Model has been extended with a ZMP optimization to improve dynamic walking stability. The case of turning during walking has also been studied and stability in this case has been enhanced through optimization. The next step of this work deals with real-time implementation of the proposed method on the prototype of SHERPA biped robot.

\section{REFERENCES}

[1] S. Ma, T. Tomiyama, and H. Wada, "Omnidirectional static walking of a quadruped robot," IEEE Transactions on Robotics, vol. 21, no. 2, pp. 152-161, 2005.

[2] M. Vukobratovic and B. Borovac, "Zero-moment point-thirty five years of its life," International Journal of Humanoid Robotics, vol. 1, no. 1, pp. 157-173, 2004.

[3] P. Sardain and G. Bessonnet, "Forces acting on a biped robot. Center of pressure-zero moment point," IEEE Transactions on Systems, Man and Cybernetics, Part A: Systems and Humans, vol. 34, no. 5, pp. 630-637, 2004.

[4] A. Goswami, "Postural stability of biped robots and the foot-rotation indicator (FRI) point," The International Journal of Robotics Research, vol. 18 , no. 6, pp. 523-533, 1999.

[5] S. Kajita, F. Kanehiro, K. Kaneko, K. Fujiwara, K. Yokoi, and H. Hirukawa, "Biped walking pattern generation by a simple threedimensional inverted pendulum model," Advanced Robotics, vol. 17, no. 2, pp. 131-147, 2003.

[6] Z. Tang and M. Er, "Humanoid 3D Gait Generation Based on Inverted Pendulum Model," in Proceedings of the IEEE 22nd International Symposium on Intelligent Control (ISIC'07), (Singapore), pp. 339-344, 2007.

[7] S. Kajita, F. Kanehiro, K. Kaneko, K. Yokoi, and H. Hirukawa, "The 3D Linear Inverted Pendulum Mode: A simple modeling for a biped walking pattern generation," in Proceedings of the IEEE/RSJ International Conference on Intelligent Robots and Systems (IROS'O1), (Maui, Hawaii, USA), pp. 239-246, 2001.

[8] S. Feng and Z. Sun, "A simple trajectory generation method for biped walking," in Proceedings of the 10th International Conference on Control, Automation, Robotics and Vision (ICARCV'08), (Hanoi, Vietnam), pp. 2078-2082, 2008

[9] S. Kajita, F. Kanehiro, K. Kaneko, K. Fujiwara, K. Harada, K. Yokoi, and $\mathrm{H}$. Hirukawa, "Biped walking pattern generation by using preview control of zero-moment point," in Proceedings of the International Conference on Robotics and Automation (ICRA'03), vol. 2, pp. 16201626, IEEE, 2003.

[10] A. Albert and W. Gerth, "Analytic Path Planning Algorithms for Bipedal Robots without a Trunk," Journal of Intelligent and Robotic Systems, vol. 36, no. 2, pp. 109-127, 2003.

[11] J. Park and K. Kim, "Biped robot walking using gravity-compensated inverted pendulum mode and computed torque control," in Proceedings of the IEEE International Conference on Robotics and Automation (ICRA'98), (Leuven, Belgium), pp. 3528-3533, 1998.

[12] J. Kanniah, Z Lwin, D. Kumar, and N. Fatt, "A ZMP management scheme for trajectory control of biped robots using a three mass model," in Proceedings of the 2nd International Conference on Autonomous Robots and Agents (ICARA'04), (Palmerston North, New Zealand), pp. 458-463, 2004

[13] T. Buschmann, S. Lohmeier, M. Bachmayer, H. Ulbrich, and F. Pfeiffer, "A collocation method for real-time walking pattern generation," Proceedings of the 2007 IEEE/RAS International Conference on Humanoid Robots (), pp. 1-6, 2007.

[14] S. Feng and Z. Sun, "Biped robot walking using three-mass linear inverted pendulum model," in Proceedings of the International Conference on Intelligent Robotics and Applications (ICIRA'08), (Wuhan, China), pp. 371-380, Springer-Verlag, 2008.

[15] I. M. C. Olaru, S. Krut, and F. Pierrot, "Novel mechanical design of biped robot sherpa using 2 dof cable differential modular joints," in Proceedings of the 2009 IEEE/RSJ International conference on Intelligent Robots and Systems (IROS'09), (St. Louis, MO, USA), pp. 4463-4468, 2009.

[16] S. Kajita, H. Hirukawa, K. Harada, and K. Yokoi, Introduction à la commande des robots humanoides. Translated in French by Sakka, S. Springer, 2009.

[17] S. Kajita, F. Kanehiro, K. Kaneko, K. Fujiwara, K. Yokoi, and H. Hirukawa, "A realtime pattern generator for biped walking," in Robotics and Automation, 2002. Proceedings. ICRA'O2. IEEE International Conference on, vol. 1, pp. 31-37, IEEE, 2002. 